\title{
Efficacy and safety profile of mucolytic/ antioxidant agents in chronic obstructive pulmonary disease: a comparative analysis across erdosteine, carbocysteine, and $\mathrm{N}$ - acetylcysteine
}

Paola Rogliani ${ }^{1 *}$ (D), Maria Gabriella Matera ${ }^{2}$, Clive Page ${ }^{3}$, Ermanno Puxeddu ${ }^{1}$, Mario Cazzola ${ }^{1}$ and Luigino Calzetta ${ }^{1}$

\begin{abstract}
Background: To date there are no head-to-head studies comparing different mucolytic/antioxidant agents. Considering the inconsistent evidence resulting from the pivotal studies on mucolytic/antioxidant agents tested in chronic obstructive pulmonary disease (COPD), and the recent publication of Reducing Exacerbations and Symptoms by Treatment with ORal Erdosteine in COPD (RESTORE) study, we have performed a meta-analysis to compare the efficacy and safety of erdosteine 600 mg/day, carbocysteine 1500 mg/day, and N-acetylcysteine (NAC) 1200 mg/day in COPD.

Methods: A pairwise and network meta-analyses were performed to assess the efficacy of erdosteine, carbocysteine, and NAC on acute exacerbation of COPD (AECOPD), duration of AECOPD, and hospitalization. The frequency of adverse events (AEs) was also investigated.

Results: Data obtained from 2753 COPD patients were extracted from 7 RCTs published between 2004 and 2017. In the pairwise meta-analysis mucolytic/antioxidant agents significantly reduced the risk of AECOPD (RR $0.7495 \% \mathrm{Cl} 0.68-0.80$ ). The network meta-analysis provided the following rank of effectiveness: erdosteine>carbocysteine>NAC. Only erdosteine reduced the risk of experiencing at least one AECOPD $(P<0.01)$ and the risk of hospitalization due to AECOPD $(P<0.05)$. Erdosteine and NAC both significantly reduced the duration of AECOPD $(P<0.01)$. The AEs induced by erdosteine, carbocysteine, and NAC were mild in severity and generally well tolerated. The quality of evidence of this quantitative synthesis is moderate.
\end{abstract}

Conclusions: The overall efficacy/safety profile of erdosteine is superior to that of both carbocysteine and NAC. Future head-to-head studies performed on the same COPD populations are needed to definitely confirm the results of this meta-analysis.

Trial registration: CRD42016053762.

Keywords: COPD, Erdosteine, Carbocysteine, N-acetylcysteine, Meta-analysis

\footnotetext{
*Correspondence: paola.rogliani@uniroma2.it

${ }^{1}$ Unit of Respiratory Medicine, Department of Experimental Medicine,

University of Rome "Tor Vergata", Via Montpellier 1, 00133 Rome, Italy

Full list of author information is available at the end of the article
}

(c) The Author(s). 2019 Open Access This article is distributed under the terms of the Creative Commons Attribution 4.0 International License (http://creativecommons.org/licenses/by/4.0/), which permits unrestricted use, distribution, and reproduction in any medium, provided you give appropriate credit to the original author(s) and the source, provide a link to the Creative Commons license, and indicate if changes were made. The Creative Commons Public Domain Dedication waiver (http://creativecommons.org/publicdomain/zero/1.0/) applies to the data made available in this article, unless otherwise stated. 


\section{Background}

The regular treatment with mucolytic/antioxidant agents such as erdosteine, carbocysteine, and $\mathrm{N}$-acetylcysteine (NAC) is recommended in patients with chronic obstructive pulmonary disease (COPD) [1]. In fact, the last Global Initiative for Chronic Obstructive Lung Disease (GOLD, 2019) document indicates that mucolytic/antioxidant drugs may reduce the risk of acute exacerbation of COPD (AECOP) and improve health status [1].

Until 2015 there was no evidence to precisely identify the target population for mucolytic/antioxidant agents in COPD [2, 3], and a recent not pre-specified post-hoc analysis PANTHEON study (Placebo-controlled study on efficAcy and safety of $\mathrm{N}$-acetylcysTeine High dose in Exacerbations of chronic Obstructive pulmoNary disease) provided conflicting evidence concerning the role of inhaled corticosteroid (ICS) therapy and smoking habit on the protective effect of high-dose NAC against the risk of AECOPD [4]. Conversely, a quantitative synthesis of randomized controlled trials (RCTs) performed in agreement with the Preferred Reporting Items for Systematic Reviews and Meta-Analyses (PRISMA) Statement demonstrated, via a meta-regression analysis, that the only factors that could significantly influence the efficacy of mucolytic/antioxidant agents in COPD were the quality of RCTs, the duration of treatment, and the number of AECOPD in the year previous the study enrolment [5]. Interestingly, the recent Reducing Exacerbations and Symptoms by Treatment with ORal Erdosteine in COPD (RESTORE) study [6] showed that erdosteine was effective in reducing the rate and duration of AECOPD irrespective of event severity and concomitant ICS treatment.

To date no head-to-head RCTs across different mucolytic/antioxidant agents have been conducted to directly compare the efficacy profile of erdosteine, carbocysteine, and NAC. Therefore, in the light of the inconsistent evidence resulting from the pivotal RCTs on mucolytic/antioxidant agents tested in COPD [7-10], and the recent publication of RESTORE study [6], we have performed a pairwise and network meta-analysis of the currently available data aimed to compare the real efficacy of erdosteine, carbocysteine, and high-dose NAC on AECOPD.

\section{Materials and methods}

\section{Search strategy}

This meta-analysis has been registered in the international database of prospectively registered systematic reviews (PROSPERO registration number: CRD42016053762), and performed in agreement with the PRISMA-P [11], with the flow diagram reported in Fig. 1A. This quantitative synthesis satisfied all the recommended items reported by the PRISMA-P checklist [11].

Two reviewers performed a comprehensive literature search for RCTs evaluating the influence of mucolytic and antioxidant agents in COPD patients. The PICO (Patient problem, Intervention, Comparison, and Outcome) framework was used to develop the literature search strategy, as previously described [12]. Namely, the "Patient problem" included subject affected by COPD;

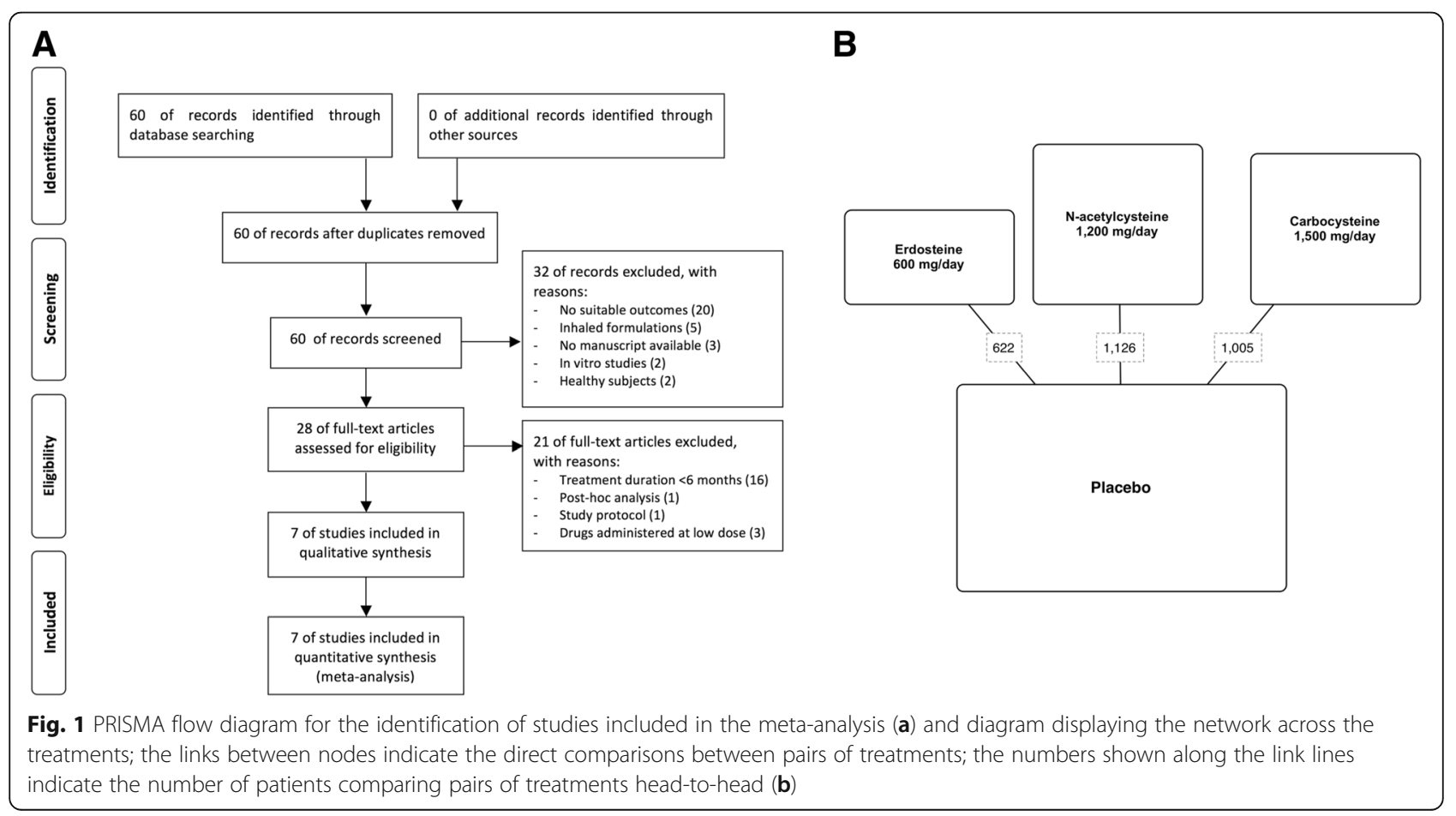


the "Intervention" regarded the administration of mucolytic and antioxidant agents; the "Comparison" was performed with regard to placebo and across each active treatment; the "Outcomes" were the duration of AECOPD, hospitalizations due to AECOPD, and adverse events (AEs).

The terms "erdosteine" OR "carbocysteine" OR "NAC" AND "COPD" AND "clinical trial" were searched in Cochrane Central Register of Controlled Trials (CENTRAL), MEDLINE, Embase, Scopus, Web of Science, ClinicalTrials.gov and EU Clinical Trials Register databases in order to provide for relevant studies available up to November 26, 2018. No language restriction was applied. The following Query Translation was used: "(("erdosteine"[Supplementary Concept] OR "erdosteine"[All Fields]) OR ("carbocysteine"[MeSH Terms] OR "carbocysteine"[All Fields]) OR ("acetylcysteine"[MeSH Terms] OR "acetylcysteine"[All Fields] OR "n acetylcysteine"[All Fields])) AND ("pulmonary disease, chronic obstructive"[MeSH Terms] OR ("pulmonary"[All Fields] AND "disease"[All Fields] AND "chronic"[All Fields] AND "obstructive"[All Fields]) OR "chronic obstructive pulmonary disease"[All Fields] OR "copd"[All Fields]) AND Clinical Trial[ptyp]".

Citations of previously published meta-analyses and relevant reviews were examined to identify further pertinent studies, if any $[3,5,13-15]$.

\section{Study selection}

Published RCTs involving COPD patients treated for more than 6 months with oral formulations of erdosteine $600 \mathrm{mg} /$ day, carbocysteine $1500 \mathrm{mg} /$ day, and NAC 1200 $\mathrm{mg} /$ day were included in this meta-analysis.

Two reviewers independently checked the relevant studies identified from literature searches obtained from the already mentioned databases. The studies were selected in agreement with the above-mentioned criteria, and any difference in opinion about eligibility was resolved by general consensus.

\section{Endpoints}

The primary endpoint of this meta-analysis was the impact of erdosteine $600 \mathrm{mg} /$ day, carbocysteine $1500 \mathrm{mg} /$ day, and NAC $1200 \mathrm{mg} /$ day on the reduction in the risk of AECOPD, compared to placebo and across each active treatment.

The secondary endpoints were the impact of erdosteine $600 \mathrm{mg} /$ day, carbocysteine $1500 \mathrm{mg} /$ day, and NAC $1200 \mathrm{mg} /$ day on the risk of experiencing at least on AECOPD, the duration of AECOPD, and the risk of hospitalization due to AECOPD, compared to placebo. The frequency of AEs was another secondary endpoint.

\section{Quality score, risk of bias and evidence profile}

The Jadad score, with a scale of 1 to 5 (score of 5 being the best quality), was used to assess the quality of the RCTs concerning the likelihood of biases related to randomization, double blinding, withdrawals and dropouts [16]. A Jadad score $\geq 3$ was defined to identify high quality studies. Two reviewers independently assessed the quality of individual studies, and any difference in opinion about the quality score was resolved by consensus.

In the pairwise meta-analysis moderate to high levels of heterogeneity between-studies were considered for $\mathrm{I}^{2}>50 \%$; the risk of publication bias was assessed for primary endpoints by applying the funnel plot and Egger's test, as previously described [16]. Evidence of asymmetry from Egger's test was considered to be significant at $P<0.1$, and the graphical representation of 90\% confidence bands have been presented [16]. The risk of bias in the network meta-analysis was checked via the consistency/inconsistency analysis to assess whether the outcomes resulting from the consistency and inconsistency models fit adequately with the line of equality, as previously described [5].

The quality of the evidence was assessed for the primary endpoint in agreement with the Grading of Recommendations Assessment, Development, and Evaluation (GRADE) system, indicating ++++ for high quality of evidence, +++ for moderate quality of evidence, ++ for low quality of evidence, and + for very low quality of evidence [17].

\section{Data extraction}

Data from included RCTs were extracted and checked for study characteristics and duration, enrolled patients, drugs and daily doses, disease characteristics, AECOPD definition, history and rate of AECOPD, age, gender, smoking habit, lung function, and Jadad score. Due to the complexity of this meta-analysis, data have been extracted in agreement with DECiMAL recommendations [18].

\section{Data analysis}

A pairwise meta-analysis was performed to quantify the impact of erdosteine $600 \mathrm{mg} /$ day, carbocysteine 1500 $\mathrm{mg} /$ day, and NAC $1200 \mathrm{mg} /$ day on primary and secondary endpoints, compared to placebo.

The follow-up duration could be not consistent across the RCTs included in this meta-analysis. Therefore, the data concerning the risk assessment have been normalized as a function of person-time (namely personseason), where one season lasts three months [19]. This method involves the conversion of the measures into a common metric (events per person-time) prior to metaanalyze the data, leading to increased estimates of effect, precision, and clinical interpretability of results [20,21]. 
Specifically, the numerator represents the count of total events and the denominator represents the given time duration multiplied by the number of patients [22]. Such a method has been supported by the Cochrane Collaboration and successfully used in recent meta-analyses [20, 21, 23, 24].

Results of the pairwise meta-analysis are expressed as relative risk (RR) or mean difference (MD), and 95\% confidence interval $(95 \% \mathrm{CI})$. Since data were selected from a series of studies performed by researchers operating independently, and a common effect size cannot be assumed, binary random-effects model was used in order to balance the study weights and adequately estimate the $95 \% \mathrm{CI}$ of the mean distribution of drugs effect on the investigated variables [16].

A network meta-analysis was also carried out to perform a comparison across the investigated agents with respect to the primary endpoint, and to rank their efficacy in reducing the risk on AECOPD. The network metaanalysis was carried out by including exclusively high quality RCTs that introduced neither heterogeneity nor bias in the overall effect estimate of primary endpoint. Since heterogeneity and bias may propagate through a network of RCTs, and thus affect the estimates differentially across regions of the network, this approach permitted to identify those studies that might alter the correct results of the network meta-analysis [25].

Full Bayesian evidence network was used was used in the network meta-analysis (chains: 4; initial values scaling: 2.5; tuning iterations: 20.000; simulation iterations: 50.000; tuning interval: 10). The convergence diagnostics for consistency and inconsistency was assessed via the Brooks-Gelman-Rubin method, as previously described [26]. Results of the network meta-analysis are expressed as relative effect (RE) and 95\% credible interval $(95 \% \mathrm{CrI})$. The probability that each intervention arm was the most effective was calculated by counting the proportion of iterations of the chain in which each intervention arm had the highest mean difference, and the surface under the cumulative ranking curve (SUCRA), representing the summary of these probabilities, was also calculated. The SUCRA is 1 when a treatment is certain to be the best, and 0 when a treatment is certain to be the worst [27].

Sensitivity analysis was performed to identify the studies that introduced heterogeneity and bias in the effect estimate of primary endpoint.

The analysis of the number needed to treat (NNT) was performed on primary endpoint. NNT is the reciprocal of the absolute risk reduction associated with an intervention over a fixed period of time [28-30]. The values of NNT are reported in this study as personbased and calculated by analyzing the Kaplan-Meier curves or by using the raw data provided in the primary publications, as previously described [31, 32]. The relative weight of each study resulting from the pairwise meta-analysis was used to calculate the weighted average rate of the investigated arms and to correctly provide NNT values.

The safety profile of the investigated treatments was investigated through a pooled analysis of the frequency of AEs, which was ranked in agreement with European Medicine Agency (EMA) guidelines: very common $\geq 1$ / 10 , common $\geq 1 / 100$ to $<1 / 10$, uncommon $\geq 1 / 1000$ to $<$ $1 / 100$, frequency not known if not calculable from the extracted data.

OpenMetaAnalyst [33] and GeMTC [34] software were used for performing the meta-analysis, GraphPad Prism (CA, US) software to graph the data, and GRADEpro GDT to assess the quality of evidence [17]. The statistical significance for the effect estimates resulting from the pairwise and network meta-analyses was assessed for $P<0.05$.

\section{Results}

\section{Studies characteristics}

Results obtained from 2753 COPD patients (11.15\% treated with erdosteine, $18.27 \%$ treated with carbocysteine, $20.41 \%$ treated with NAC, and $50.16 \%$ treated with placebo) were selected from 7 RCTs $[6-10,35,36]$ published between 2004 and 2017. The relevant characteristics of studies, disease, and patients and the definition of AECOPD are described in Table 1; Fig. 1B shows the network across the treatments.

Five RCTs were published as full-text papers [6-10], and two RCTs as letters to the editor [35, 36]. Six RCTs were published as high quality studies (Jadad score $\geq 3$ ) $[6-10,36]$, and one as low-quality study (Jadad score $=1$ ) [35]. The duration of treatment ranged from 32 weeks to 52 weeks.

\section{Primary endpoint}

The pairwise meta-analysis indicated that erdosteine, carbocysteine, and NAC both significantly reduced the risk of AECOPD. The overall effect estimate was affected by high and significant heterogeneity that was driven by the studies on carbocysteine and NAC. Conversely, the effect estimate resulting for erdosteine was free from any heterogeneity (Fig. 2A).

The sensitivity analysis indicated that the main source of heterogeneity was introduced by the studies of Tatsumi et al. and Yasuda et al. on carbocysteine [35, 36], as excluding these RCTs the overall heterogeneity reduced at acceptable and not significant levels $\left(\mathrm{I}^{2} 26 \%, P>0.05\right)$ (Fig. 2B). Nevertheless, the visual inspection of funnel plot indicated that the study of Tse et al. [8] introduced a certain level of publication bias (Fig. 2C). In fact Egger's test indicated that the results of sensitivity analysis were affected by significant publication bias, as the 


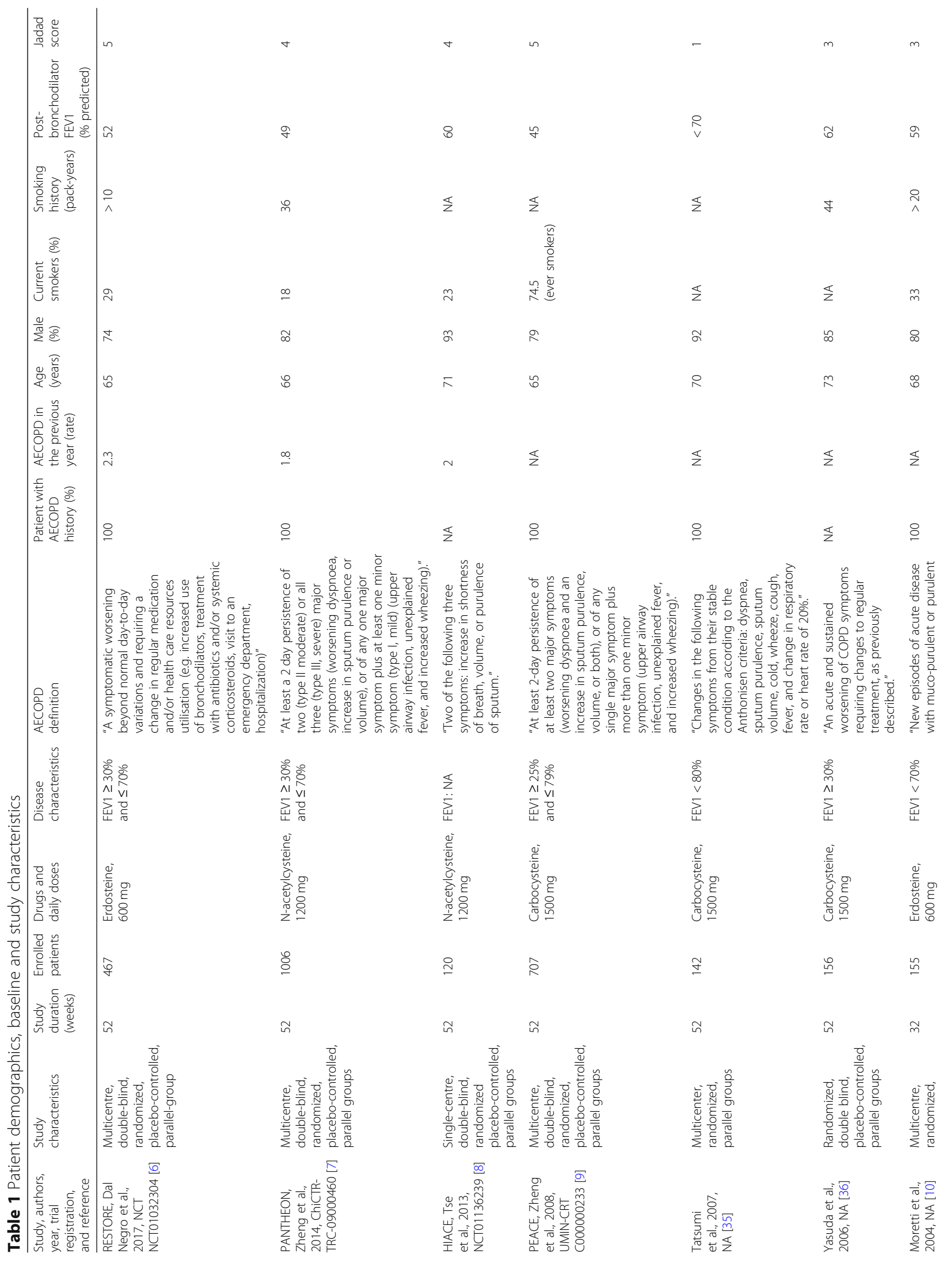




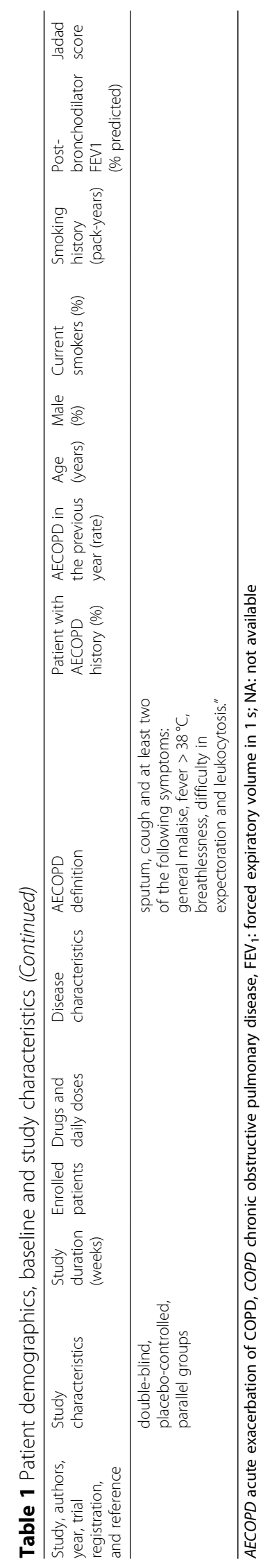




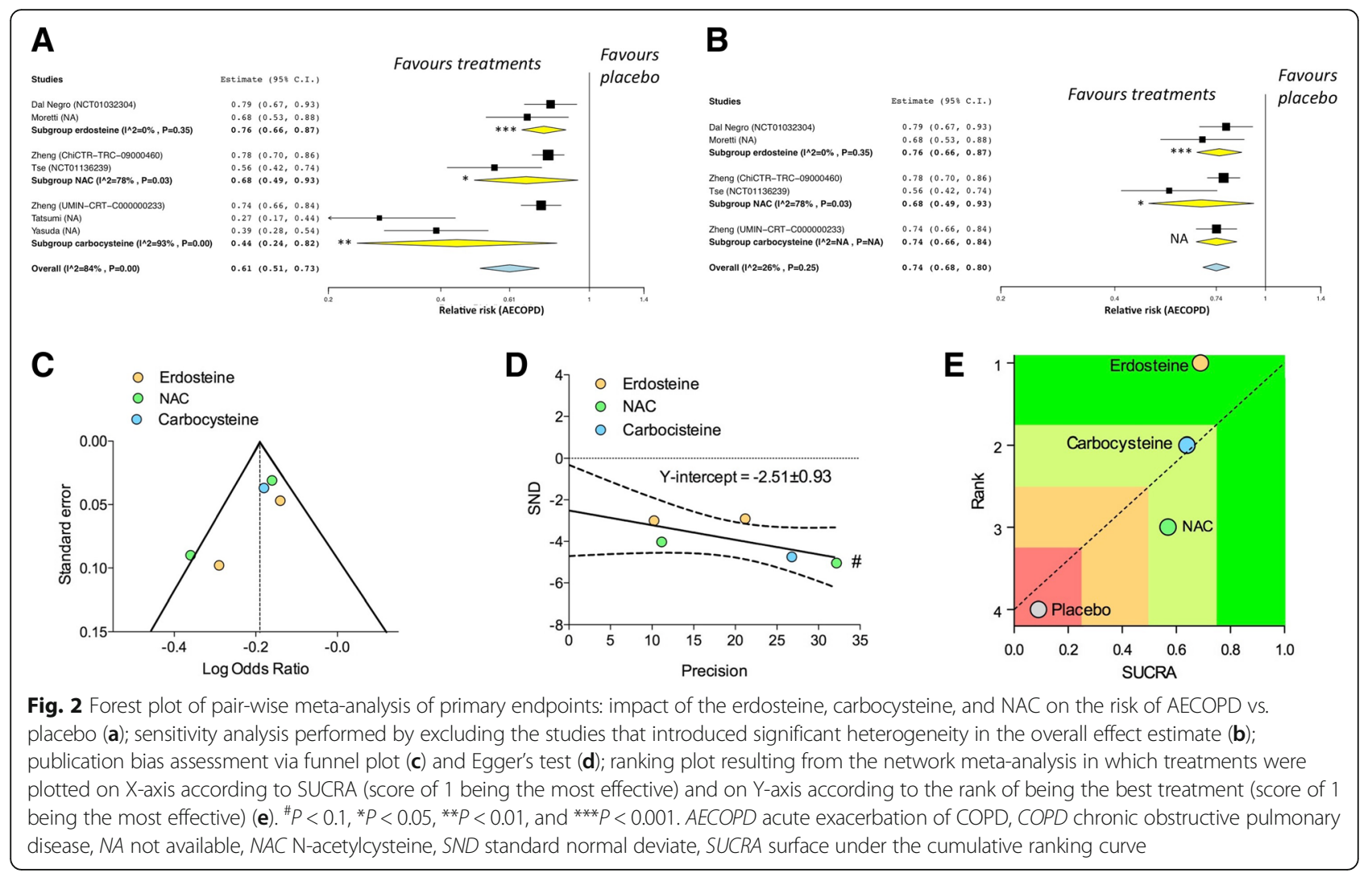

regression line and 90\% confidence bands did not intercept the origin of the graph (Fig. 2D). A further sensitivity analysis confirmed that the study of Tse et al. [8] was a source of publication bias, as the regression line and 90\% confidence bands of Egger's test intercepted the origin of the graph (Y-intercept $-1.64,-6.23$ to 3.00 ) when this RCT [8] was removed from the analysis.

The results of the network meta-analysis showed that there was not significant difference $(P>0.05)$ across the investigated drugs with respect to their effect against the risk of AECOPD (erdosteine vs. carbocysteine: RE 0.97, 95\% CrI 0.34-2.56; carbocysteine vs. NAC: RE 0.95, 95\%CrI 0.29-2.92; erdosteine vs. NAC: RE 0.92, 95\%CrI $0.32-2.38$ ). However, the network meta-analysis indicated that erdosteine was the most effective agent in preventing the risk of AECOPD (upper quartile in the SUCRA ranking), followed by carbocysteine and NAC (third quartile in the SUCRA ranking) (Fig. 2E).

The consistency/inconsistency analysis showed that all points fit adequately with the line of equality (goodness of fit: $\mathrm{R}^{2}$ 0.99; slope $0.96,95 \% \mathrm{CI} 0.90-1.02$ ), indicating that the network meta-analysis was not affected by significant bias.

The person-based NNT analysis reported that 10.11 (95\%CI 5.41-76.39) patients had to be treated with erdoisteine for one year to prevent one AECOPD, compared to placebo $(P<0.05)$. Conversely, the NNT values for both carbocysteine $(30.92,95 \% \mathrm{CI} 8.61-\infty)$ and NAC $(15.69,95 \%$ CI $7.31-\infty)$ were not significantly different $(P>0.05)$ than placebo.

The GRADE analysis indicated high quality of evidence $(++++)$ for erdosteine, low quality of evidence $(+$ $+)$ for carbocysteine, and moderate quality of evidence $(+++)$ for NAC, when compared to placebo. The quality of evidence in the network meta-analysis comparing erdosteine, carbocysteine, and NAC via placebo arm was moderate $(+++)$.

\section{Secondary endpoints}

Erdosteine, but neither carbocysteine nor NAC, significantly $(P<0.01)$ reduce the risk of experiencing at least one AECOPD, compared to placebo (Fig. 3A). Erdosteine and NAC both significantly $(\mathrm{P}<0.01$ and $P<0.001$, respectively) reduced the duration of $\mathrm{AECOPD}$, compared to placebo; no data on this outcome are currently available for carbocysteine (Fig. 3B). Erdosteine, but not NAC, significantly $(P<0.05)$ reduced the risk of hospitalization due to AECOPD, compared to placebo; no data on this outcome are currently available for carbocysteine (Fig. 3C).

The pooled analysis of safety profile showed that AEs were very common in patients treated with NAC, and common in those treated with either erdosteine and carbocysteine, or that received placebo. However, AEs were 

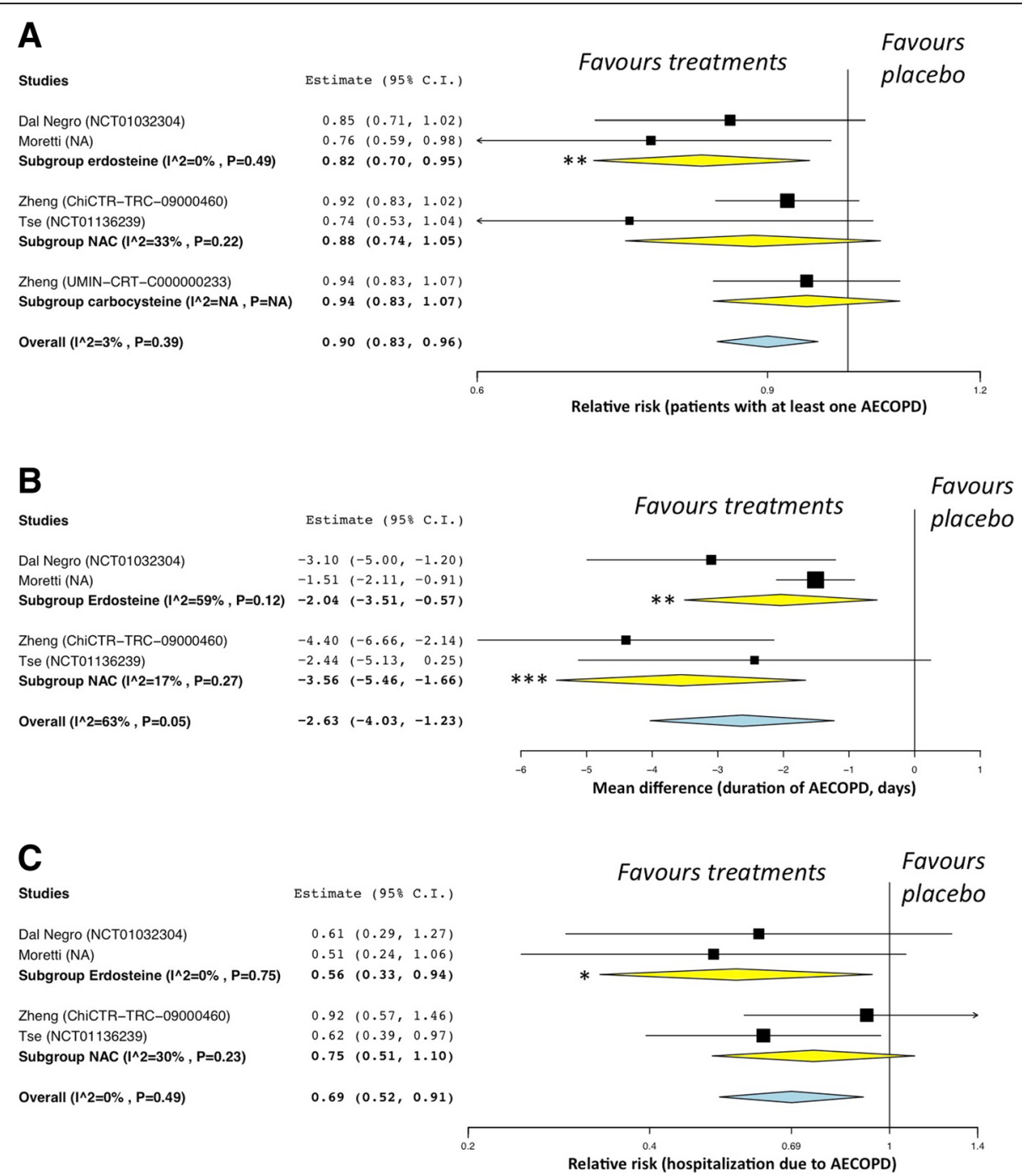

Fig. 3 Forest plot of pair-wise meta-analysis of secondary endpoints: impact of the erdosteine, carbocysteine, and NAC on the risk of experiencing at least one AECOPD (a), duration of AECOPD (b), and risk of hospitalization due to AECOPD (c), vs. placebo. ${ }^{*} P<0.05$ and ${ }^{* *} P<0.01$. AECOPD: acute exacerbation of COPD; COPD: chronic obstructive pulmonary disease

mild in severity and generally well tolerated. The most frequent AEs was respiratory tract infection (NAC: $10.85 \%$, carbocysteine: $0.56 \%$, erdosteine: not detectable), and detailed frequencies of further specific AEs are reported in Table 2.

\section{Discussion}

In this quantitative synthesis of current literature both erdosteine, carbocysteine, and NAC fulfilled the primary endpoint represented by the reduction in the risk of AECOPD. Considering exclusively the high-quality RCTs that did not introduce significant heterogeneity in the pairwise meta-analysis, this study indicates that the mean effect estimate of the overall impact of mucolytic/antioxidant agents reached the minimal clinically important difference (MCID: $\approx 0.75 \mathrm{RR}$ ) $[19,38]$ in reducing the risk of
AECOPD compared to placebo. In any case, the results of pairwise meta-analysis seem to be affected by a certain level of publication bias that was mainly related with the results extracted from the study of Tse et al. [8], as confirmed by both funnel plot and Egger's test analyses.

Although we found no significant difference across the investigated drugs with respect to their protective effect against AECOPD, the SUCRA analysis resulting from the network meta-analysis indicated that erdosteine was the most effective agent, followed by carbocysteine and NAC. Interestingly, the consistency/inconsistency analysis showed that the network meta-analysis was not affected by significant bias.

The superiority of erdosteine with respect to carbocysteine and NAC was also confirmed by the NNT analysis, that provided significant NNT values for erdosteine but 
Table 2 Pooled analysis of AEs extracted from the studies on erdosteine, carbocysteine, and NAC in COPD patients and ranked by frequency in agreement with EMA guidelines [37]

\begin{tabular}{|c|c|c|c|c|}
\hline & Erdosteine & Carbocysteine & NAC & Placebo \\
\hline Total number of subjects & 354 & 557 & 553 & 1151 \\
\hline Frequency (\%) of all AEs & $1.32(+++)$ & $2.26(+++)$ & $18.26(++++)$ & $8.43(+++)$ \\
\hline \multicolumn{5}{|l|}{ Frequency (\%) of specific AEs: } \\
\hline respiratory tract infection & ND & $0.56(+++)$ & $10.85(++++)$ & $4.26(+++)$ \\
\hline gastrointestinal disorders & $0.44(++)$ & $1.13(+++)$ & $4.16(+++)$ & $4.26(+++)$ \\
\hline pruritus & ND & ND & $1.08(+++)$ & $2.69(+++)$ \\
\hline cerebrovascular disorders & $0.44(++)$ & ND & $0.90(++)$ & $0.09(+)$ \\
\hline dizziness & ND & ND & $0.72(++)$ & $0.09(+)$ \\
\hline musculoskeletal disorders & ND & $0.28(++)$ & $0.54(++)$ & $0.78(++)$ \\
\hline hepatobiliary disorders & $0.44(++)$ & ND & ND & ND \\
\hline malaise & ND & $0.28(++)$ & ND & $0.09(+)$ \\
\hline insomnia & ND & ND & ND & $0.26(++)$ \\
\hline increased cough & ND & ND & ND & $0.17(++)$ \\
\hline
\end{tabular}

++++ : very common $(\geq 1 / 10)$; +++: common $(\geq 1 / 100$ to $<1 / 10)$; ++ : uncommon $(\geq 1 / 1000$ to $<1 / 100)$; + : rare $(\geq 1 / 10,000$ to $<1 / 1000)$; AEs: adverse events; COPD: chronic obstructive pulmonary disease; EMA: European Medicine Agency; NAC: N-acetylcysteine; ND: not detectable (frequency not known)

neither for carbocysteine nor for NAC, when compared to placebo. Specifically, $\approx 10$ patients had to be treated with erdoisteine for one year to prevent one AECOPD.

The analysis of the quality of evidence concerning the efficacy on risk of AECOPD compared to placebo indicates that we can be very confident that the true effect of erdosteine lies close to that of the estimate of the effect. Conversely, moderate to limited confidence resulted for NAC and carbocysteine, respectively. This means that while further research is very unlikely to change our confidence in the estimate of effect resulting for erdosteine, future studies are likely to very likely to have an important impact on the confidence in the estimate of effect resulting for NAC and carbocysteine [39]. Due to the indirect comparison across erdosteine, carbocysteine, and NAC in the Bayesian process, the quality of evidence of network meta-analysis is moderate, thus representing the main limitation of our study.

Further limits of this quantitative synthesis are represented by the difference, or missing data, concerning the baseline characteristics of COPD patients enrolled in the RCTs and included in the meta-analysis, namely the current smoking levels, respiratory function impairment, and rate of AECOPD in the previous year. Besides, also the definition of AECOPD was not consistent across the studies included in this meta-analysis. The risk assessment was normalized as a function of person-time since the follow-up was not consistent across the RCTs included in the meta-analysis. Although this procedure has been extensively validated and supported by the Cochrane Collaboration [20, 21], adjusting data for different follow-up duration may represent another minor limitation of the study.
Concerning the secondary endpoints, only erdosteine was significantly effective in reducing the risk of experiencing at least one AECOPD and the risk of hospitalization due to AECOPD, whereas the duration of AECOPD was significantly reduced by both erdosteine and NAC.

The pooled analysis of AEs indicates that the mucolytic/ antioxidant agents investigated in this study are characterized by a positive safety profile, and that the recorded AEs were mild in severity and generally well tolerated.

Indeed the efficacy and safety profile of mucolytic/antioxidant agents resulting by quantitative synthesis of the current literature supports the use of erdosteine, carbocysteine, and NAC in COPD patients, as recommended by the last (GOLD) document [1]. Considering that most the pivotal RCTs $[6,7,9]$ explored the impact of mucolytic/ antioxidant agents on the rate of overall AECOPD, that includes both mild, moderate and severe exacerbations, to date it is still unclear whether these drugs can be effective in specifically reducing the risk of moderate or severe AECOPD. Furthermore, considering the moderate quality of evidence resulting from the network meta-analysis, and the lack of significant difference across the investigated drugs, we cannot exclude that future study may change the rank provided by the SUCRA analysis.

\section{Conclusion}

Concluding, the current evidence suggests that the overall efficacy/safety profile of erdosteine is superior to that of both carbocysteine and NAC. However, future head-tohead studies performed on the same COPD populations are needed to definitely confirm the results of this quantitative synthesis. 


\section{Abbreviations}

AECOPD: acute exacerbation of COPD; CENTRAL: Cochrane Central Register of Controlled Trials; Cl: confidence interval; COPD: chronic obstructive pulmonary disease; Crl: credible interval; EMA: European Medicine Agency; GOLD: Global Initiative for Chronic Obstructive Lung Disease; GRADE: Grading of Recommendations Assessment, Development, and Evaluation; ICS: inhaled corticosteroid; MCID: minimal clinically important difference; MD: mean difference; NAC: N-acetylcysteine; NNT: number needed to treat; PANTHEON: Placebo-controlled study on efficAcy and safety of N-acetylcysTeine High dose in Exacerbations of chronic Obstructive pulmoNary disease; PICO: Patient problem, Intervention, Comparison, and Outcome; PRISMA: Preferred Reporting Items for Systematic Reviews and Meta-Analyses; $R C T$ : randomized controlled trial; RE: relative effect; RESTORE: Reducing Exacerbations and Symptoms by Treatment with ORal Erdosteine; RR: relative risk; SUCRA: surface under the cumulative ranking curve

\section{Acknowledgements}

NA

\section{Authors' contributions}

PR, MGM $, C P, E P, M C$ and LC have made substantial contributions to conception and design, acquisition of data, and analysis and interpretation of data; were involved in drafting the manuscript or revising it critically for important intellectual content; gave final approval of the version to be published. Each author participated sufficiently in the work to take public responsibility for appropriate portions of the content; and agreed to be accountable for all aspects of the work in ensuring that questions related to the accuracy or integrity of any part of the work are appropriately investigated and resolved.

\section{Funding}

Edmond Pharma Srl (Paderno Dugnano, Milan, Italy) has provided unconditional support and funding in this research.

\section{Availability of data and materials}

The datasets used and/or analysed during the current study are available from the corresponding author on reasonable request.

\section{Ethics approval and consent to participate}

Not applicable.

\section{Consent for publication}

Not applicable.

\section{Competing interests}

PR participated as a lecturer, speaker, and advisor in scientific meetings and courses under the sponsorship of Almirall, AstraZeneca, Biofutura, Boehringer Ingelheim, Chiesi Farmaceutici, GlaxoSmithKline, Menarini Group, Mundipharma, and Novartis. Her department was funded by Almirall, Boehringer Ingelheim, Novartis, Zambon and Chiesi Farmaceutici. MGM has participated as a lecturer, speaker, and advisor in scientific meetings and courses under the sponsorship of Almirall, AstraZeneca, Boehringer Ingelheim, Chiesi Farmaceutici, GlaxoSmithKline and Novartis, and has been a consultant to Chiesi Farmaceutici.

$\mathrm{CP}$ has acted as a Consultant for Recipharma, ImmunoRegulation, PrEP Biopharma, Ockham Biosciences and Eurodrug. CP also owns equity in Verona Pharma who are developing novel drugs for the treatment of respiratory diseases. EP has no conflict of interest to declare.

$M C$ has participated as a lecturer, speaker, and advisor in scientific meetings and courses under the sponsorship of Almirall, AstraZeneca, Biofutura, Boehringer Ingelheim, Chiesi Farmaceutici, GlaxoSmithKline, Menarini Group, Lallemand, Mundipharma, Novartis, Pfizer, Verona Pharma, and Zambon, and has been a consultant to ABC Farmaceutici, Recipharm, Chiesi Farmaceutici, Lallemand, Novartis, Verona Pharma, and Zambon. His department was funded by Almirall, Boehringer Ingelheim, Novartis, and Zambon. $L C$ has participated as advisor in scientific meetings under the sponsorship of Boehringer Ingelheim and Novartis, received non-financial support by AstraZeneca, received a research grant partially funded by Chiesi Farmaceutici, Boehringer Ingelheim, Novartis, and Almirall, and is or has been a consultant to ABC Farmaceutici, Recipharm, Zambon, Verona Pharma, and Ockham Biotech. His department was funded by Almirall, Boehringer Ingelheim, Novartis, Zambon and Chiesi Farmaceutici.

\section{Author details}

${ }^{1}$ Unit of Respiratory Medicine, Department of Experimental Medicine, University of Rome "Tor Vergata", Via Montpellier 1, 00133 Rome, Italy. unit of Pharmacology, Department of Experimental Medicine, University of Campania "Luigi Vanvitelli", Naples, Italy. "Sackler Institute of Pulmonary Pharmacology, Institute of Pharmaceutical Science, King's College London, London, UK.

Received: 16 April 2019 Accepted: 20 May 2019

Published online: 27 May 2019

\section{References}

1. GOLD: Global Initiative for Chronic Obstructive Lung Disease. Global strategy for diagnosis, management, and prevention of COPD - 2019 Report. Available from https://goldcopd.org/wp-content/uploads/2018/11/ GOLD-2019-v1.5-FINAL-04Nov2018_WMS.pdf. Last accessed March 11, 2019. 2019.

2. Poole P, Chong J, Cates CJ. Mucolytic agents versus placebo for chronic bronchitis or chronic obstructive pulmonary disease. Cochrane Database Syst Rev. 2015:CD001287.

3. Cazzola M, Calzetta L, Page C, Jardim J, Chuchalin AG, Rogliani P, Gabriella Matera M. Influence of $\mathrm{N}$-acetylcysteine on chronic bronchitis or COPD exacerbations: a meta-analysis. Eur Respir Rev. 2015;24:451-61.

4. Papi A, Zheng J, Criner GJ, Fabbri LM, Calverley PMA. Impact of smoking status and concomitant medications on the effect of high-dose $\mathrm{N}$ acetylcysteine on chronic obstructive pulmonary disease exacerbations: a post-hoc analysis of the PANTHEON study. Respir Med. 2019;147:37-43.

5. Cazzola M, Rogliani P, Calzetta L, Hanania NA, Matera MG. Impact of mucolytic agents on COPD exacerbations: a pair-wise and network metaanalysis. COPD. 2017;14:552-63.

6. Dal Negro RW, Wedzicha JA, Iversen M, Fontana G, Page C, Cicero AF, Pozzi E, Calverley PMA, Group R, Study R. Effect of erdosteine on the rate and duration of COPD exacerbations: the RESTORE study. Eur Respir J. 2017;50.

7. Zheng JP, Wen FQ, Bai CX, Wan HY, Kang J, Chen P, Yao WZ, Ma LJ, Li X, Raiteri $L$, et al. Twice daily $\mathrm{N}$-acetylcysteine $600 \mathrm{mg}$ for exacerbations of chronic obstructive pulmonary disease (PANTHEON): a randomised, doubleblind placebo-controlled trial. Lancet Respir Med. 2014;2:187-94.

8. Tse HN, Raiteri L, Wong KY, Yee KS, Ng LY, Wai KY, Loo CK, Chan MH. Highdose $\mathrm{N}$-acetylcysteine in stable COPD: the 1-year, double-blind, randomized, placebo-controlled HIACE study. Chest. 2013;144:106-18.

9. Zheng JP, Kang J, Huang SG, Chen P, Yao WZ, Yang L, Bai CX, Wang CZ, Wang C, Chen BY, et al. Effect of carbocisteine on acute exacerbation of chronic obstructive pulmonary disease (PEACE study): a randomised placebo-controlled study. Lancet. 2008;371:2013-8.

10. Moretti M, Bottrighi P, Dallari R, Da Porto R, Dolcetti A, Grandi P, Garuti G, Guffanti E, Roversi P, De Gugliemo M, et al. The effect of long-term treatment with erdosteine on chronic obstructive pulmonary disease: the EQUALIFE study. Drugs Exp Clin Res. 2004;30:143-52.

11. Moher D, Shamseer L, Clarke M, Ghersi D, Liberati A, Petticrew M, Shekelle P, Stewart LA, Group P-P. Preferred reporting items for systematic review and meta-analysis protocols (PRISMA-P) 2015 statement. Syst Rev. 2015:4:1.

12. Schardt C, Adams MB, Owens T, Keitz S, Fontelo P. Utilization of the PICO framework to improve searching PubMed for clinical questions. BMC Med Inform Decis Mak. 2007;7:16.

13. Cazzola M, Calzetta L, Page C, Rogliani P, Matera MG. Impact of erdosteine on chronic bronchitis and COPD: a meta-analysis. Pulm Pharmacol Ther. 2018:48:185-94.

14. Zeng Z, Yang D, Huang X, Xiao Z. Effect of carbocisteine on patients with COPD: a systematic review and meta-analysis. Int J Chron Obstruct Pulmon Dis. 2017;12:2277-83.

15. Fowdar K, Chen H, He Z, Zhang J, Zhong X, Zhang J, Li M, Bai J. The effect of $\mathrm{N}$-acetylcysteine on exacerbations of chronic obstructive pulmonary disease: a meta-analysis and systematic review. Heart Lung. 2017;46:120-8.

16. Calzetta L, Rogliani P, Matera MG, Cazzola M. A systematic review with meta-analysis of dual bronchodilation with LAMA/LABA for the treatment of stable COPD. Chest. 2016;149:1181-96.

17. Guyatt G, Oxman AD, Akl EA, Kunz R, Vist G, Brozek J, Norris S, Falck-Ytter Y, Glasziou P, DeBeer $H$, et al. GRADE guidelines: 1. Introduction-GRADE evidence profiles and summary of findings tables. J Clin Epidemiol. 2011;64: 383-94. 
18. Pedder H, Sarri G, Keeney E, Nunes V, Dias S. Data extraction for complex meta-analysis (DECiMAL) guide. Syst Rev. 2016;5:212.

19. Calzetta L, Matera MG, Braido F, Contoli M, Corsico A, Di Marco F, Santus P, Scichilone N, Cazzola M, Rogliani P. Withdrawal of inhaled corticosteroids in COPD: a meta-analysis. Pulm Pharmacol Ther. 2017;45:148-58.

20. Guevara JP, Berlin JA, Wolf FM. Meta-analytic methods for pooling rates when follow-up duration varies: a case study. BMC Med Res Methodol. 2004:4:17.

21. Higgins JPT, Green S: Cochrane Handbook for Systematic Reviews of Interventions Version 5.1.0 [updated March 2011]. Available from https:// handbook-5-1.cochrane.org/chapter_9/9_6_4_meta_regression.htm. The Cochrane Collaboration 2011, 9.6.4 Meta-regression.

22. Cazzola M, Calzetta L, Rogliani P, Page C, Matera MG. Impact of doxofylline in COPD: a pair-wise meta-analysis. Pulm Pharmacol Ther. 2018.

23. Cazzola M, Calzetta L, Barnes PJ, Criner GJ, Martinez FJ, Papi A, Gabriella Matera M. Efficacy and safety profile of xanthines in COPD: a network metaanalysis. Eur Respir Rev. 2018;27.

24. Calzetta L, Ora J, Cavalli F, Rogliani P, O'Donnell DE, Cazzola M. Impact of LABA/LAMA combination on exercise endurance and lung hyperinflation in COPD: a pair-wise and network meta-analysis. Respir Med. 2017:129:189-98.

25. Li T, Puhan MA, Vedula SS, Singh S, Dickersin K. Ad hoc network meta-analysis methods meeting working G: network meta-analysis-highly attractive but more methodological research is needed. BMC Med. 2011;9:79.

26. Calzetta L, Rogliani P, Ora J, Puxeddu E, Cazzola M, Gabriella Matera M. LABA/LAMA combination in COPD: a meta-analysis on the duration of treatment. Eur Respir Rev. 2016;0:1-11. https://doi.org/10.1183/16000617. 0043-2016.

27. Cazzola M, Calzetta L, Rogliani P, Matera MG. Tiotropium formulations and safety: a network meta-analysis. Therapeutic Advances in Drug Safety. 2016: 2042098616667304. https://doi.org/10.1177/2042098616667304.

28. Cazzola M. Application of number needed to treat (NNT) as a measure of treatment effect in respiratory medicine. Treat Respir Med. 2006;5:79-84.

29. Aaron SD, Fergusson DA. Exaggeration of treatment benefits using the "event-based" number needed to treat. Can Med Assoc J. 2008;179:669-71.

30. Suissa S. Number needed to treat in COPD: exacerbations versus pneumonias. Thorax. 2013;68:540-3.

31. Altman DG, Andersen PK. Calculating the number needed to treat for trials where the outcome is time to an event. BMJ. 1999;319:1492-5.

32. Altman DG. Confidence intervals for the number needed to treat. BMJ. 1998;317:1309-12.

33. Wallace BC, Dahabreh IJ, Trikalinos TA, Lau J, Trow P, Schmid CH. Closing the gap between methodologists and end-users: $R$ as a computational Back-end. J Stat Softw. 2012;49:1-15.

34. van Valkenhoef $G$, Lu G, de Brock B, Hillege $H$, Ades $A E$, Welton NJ. Automating network meta-analysis. Res Synth Methods. 2012;3:285-99.

35. Tatsumi K, Fukuchi Y, Group PS. Carbocisteine improves quality of life in patients with chronic obstructive pulmonary disease. J Am Geriatr Soc. 2007:55:1884-6.

36. Yasuda H, Yamaya M, Sasaki T, Inoue D, Nakayama K, Tomita N, Yoshida M, Sasaki H. Carbocisteine reduces frequency of common colds and exacerbations in patients with chronic obstructive pulmonary disease. J Am Geriatr Soc. 2006;54:378-80.

37. European Commission. A guideline on summary of product characteristics (SmPC). Available at http://ec.europa.eu/health//sites/health/files/files/eudralex/ vol-2/c/smpc_guideline_rev2_en.pdf. Accessed June 22, 2017. 2009.

38. Cazzola M, Rogliani P, Calzetta L, Matera MG. Triple therapy versus single and dual long-acting bronchodilator therapy in COPD: a systematic review and meta-analysis. Eur Respir J. 2018;52.

39. Balshem $H$, Helfand $M$, Schunemann HJ, Oxman AD, Kunz R, Brozek J, Vist GE, Falck-Ytter Y, Meerpohl J, Norris S, Guyatt GH. GRADE guidelines: 3 Rating the quality of evidence. J Clin Epidemiol. 2011:64:401-6.

\section{Publisher's Note}

Springer Nature remains neutral with regard to jurisdictional claims in published maps and institutional affiliations.

Ready to submit your research? Choose BMC and benefit from:

- fast, convenient online submission

- thorough peer review by experienced researchers in your field

- rapid publication on acceptance

- support for research data, including large and complex data types

- gold Open Access which fosters wider collaboration and increased citations

- maximum visibility for your research: over $100 \mathrm{M}$ website views per year

At BMC, research is always in progress.

Learn more biomedcentral.com/submissions 